Administración de Empresas 


\title{
El sistema económico y producti- vo colombiano, transformaciones y cambios de cara a las Tecnologías de la Información y Comunicación (TIC)
}

\begin{abstract}
The economic and productive Colombian system, transformations and changes face to Information and Communications Technology (ICT)
\end{abstract}

Sonia Esmeralda Rojas Rojas* María Odilia Jiménez Ricaurte**

Artículo de investigación

Recepción: 18 de octubre de 2019

Aceptación: 26 de febrero de 2020

Como citar este artículo

Rojas, S y Jiménez, M. (2019). El sistema económico y productivo colombiano, transformaciones y cambios de cara a las Tecnologías de la Información y la Comunicación (TIC). Revista Colombiana de Ciencias Administrativas 1(2), pp. 23-39.

Recuperado a partir de: http://cipres.sanmateo.edu.co/index.php/rcca

\footnotetext{
*Doctora en Gerencia Pública y Política Social, de la Universidad de Baja California (México), Magister en Administración Pública de la Escuela Superior de Administración Pública -ESAP-, especialista en Administración en Salud con Énfasis en Seguridad Social de la Pontifica Universidad Javeriana y Bioquímica egresada de la Universidad Antonio Nariño. Investigadora institucional en la Fundación Universitaria San Mateo. Correo electrónico: serojas@ sanmateo.edu.co 


\section{Resumen}

En Colombia, el sistema económico está conformado por dos (2) sectores, a saber: el público y el privado. El primero tiene como función lograr el bienestar de la sociedad; el segundo, el incesante lucro. Aunque se encuentren en polos opuestos, por sus funciones disímiles, al complementarse entre sí, estos dos (2) sectores forjan la verdadera riqueza de un país; son los encargados de generar el crecimiento económico y el desarrollo social del mismo. El crecimiento y desarrollo tienen que sostenerse sobre sólidas bases de investigación e innovación, como presenta este artículo. En esto el Gobierno, en cabeza de COLCIENCIAS, es decir, la academia y el sector empresarial, juegan un papel preponderante. La investigación e innovación, que, a partir de la globalización, ha forjado grandes cambios en los procesos productivos; el principal de ellos: transitar de la producción en serie a la producción flexible. Esto, ha generado que el entorno (en donde se encuentran las organizaciones) se vuelva cambiante y exigente, desde el momento cero (0). El resultado son organizaciones más flexibles, y con requerimientos; en cuanto a mano de obra, más exigente.

Palabras claves: Globalización; producción; organizaciones; competitividad; TIC.

\section{Abstract:}

In Colombia, the economic system is conform by two (2) sectors: the public and private. The first one has to achieve the society wellness; the second one, the incessant lucre. Although the public and private sector are in poles apart for their dissimiles functions, when their complement each other, this two (2) sectors forge the true wealth of a country; they generate the economic growth and the social development of each country. The growth and the development have to sustain over solid investigation and innovation bases; the aim of this paper is to do so. About the last idea, the government, with COLCIENCIAS at the forefront, i.e. the academia and the business sector, play a preponderant role. The investigation and innovation that, from globalization, have forged big changes in the productive process; the first of all, transit from mass production to flexible production. This has generated the environment (where it is find the organizations) as a changing and demanding, from the zero moment. The results show more flexible organizations with more requirements; as to the workforce, more demanding. 
Keywords: Globalization; production; organizations; competitivity; ICT.

\section{Introducción}

El presente documento es uno de los productos de la investigación Cambios Estructurales de la Administración de Empresas a partir del modelo aperturista. Es una investigación formativa, de tipo documental; su estrategia metodológica es la indagación. Cuenta con la colaboración de estudiantes ayudantes del programa de Administración de Empresas, de la Fundación Universitaria San Mateo; a partir de la exploración documental, recopilaron información la cual se analiza por medio de la reflexión. De ahí que el carácter del documento se inclina hacia la opinión.

La pretensión de la investigación es reflexionar de qué manera el modelo aperturista ha influido en la estructura de la administración de empresas, como ciencia. Se parte del lazo relacional existente entre lo económico, lo productivo, la gestión empresarial y la academia (Administración de Empresas). Entonces, temas atinentes al sistema económico y productivo colombiano han sido de los principales en reflexionar; más si se tiene en cuenta que, a partir del modelo aperturista iniciado en la década de 1990, estos fueron los que más afectados por la entrada a una era económica caracterizada por la apertura al comercio exterior.

El cambio de modelo económico hizo temblar a las estructuras productivas del país; colocó en jaque a muchas empresas, en especial a las del sector industrial, que vieron como la no adopción a los cambios las colocaba al borde del exterminio. Así, la adopción se volvió la estrategia a trabajar por las empresas para enfrentar el fenómeno provocado por la apertura: la competitividad. Una competitividad marcada por las tecnologías de la información, las cuales no solo llegaron para quedarse, también implementaron nuevas reglas de juego, tanto en el ámbito empresarial como en el académico.

Las Tecnologías de la Información y la Comunicación (TIC) lograron volver dinámico, cambiante y retador (peligroso dirían algunos) al entorno. Esto no solo para las empresas sino para su modelo de gestión, el cual ha venido cambiando de manera camaleónica conforme el modelo económico se cimienta y la estrategia empresarial evoluciona. Lo anterior esclarece al lector el abordaje dado a este documento de opinión, el cual gira entorno al sistema económico y productivo colombiano, y a la manera como las TIC han venido a ser parte protagónica de su transformación. 


\section{Sistema económico colombiano}

En Colombia, el sistema económico manejado y establecido es el capitalismo mixto; se caracteriza porque en su interior confluyen dos (2) sectores: el público, conformado por un conjunto de instituciones, empresas, poderes y organismos administrativos; el Estado tiene como principal función, cumplir y hacer la política y la voluntad expresadas en las leyes que hay en el país. Este sector, en el que confluyen instituciones públicas que producen o suministran los servicios colectivos (educación, salud, justicia, defensa, por nombrar algunos), es financiado por aquellos recursos que llegan a través de los impuestos y contribuciones obligatorias. Los recursos hacen parte del denominado tesoro público. Ha de entenderse que la finalidad del sector público no es el lucro (bien particular), sino el bienestar de la sociedad (bien general).

Por su parte el sector privado, a diferencia del sector público, busca el lucro en su actividad. No es controlado, en cambio es regulado por el Estado; tiene como prioridad lograr la transformación productiva, industrial y comercial del país. Como bien lo dice La República (2019): "El compromiso del sector privado no se limita simplemente al ejercicio productivo", "[...] siempre ha estado marcado por la premisa de "ir más allá", por medio de una mirada integral que contribuye a la solución de problemas estructurales con incidencia directa en el avance social y ambiental del país". En estos términos, el compromiso del sector privado debe ir siempre encaminado al progreso, mejora y avance de la economía del país (Mojica Montoya, 2018).

Aunado a lo anterior, hay que decir que la Constitución Política de Colombia de 1991, en su artículo 58, manifiestan que "la propiedad es una función social que implica obligaciones". En el artículo 333, dice: "la actividad económica y la iniciativa privada son libres dentro del bien común. La libre competencia económica es un derecho de todos los entes económicos que supone responsabilidades". Es claro que constitucionalmente se propende por la garantía de la propiedad privada, por la libre competencia, y por la inalienabilidad de los derechos adquiridos con arreglo a las leyes civiles.

Aparte de los sectores público y privado, dentro del sistema económico colombiano confluye la economía social, constituida por "cooperativas, empresas de trabajo asociado, sociedades laborales, organizaciones no lucrativas, asociaciones caritativas, mutuas o mutualidad y microemprendimientos asociativos" (FAMUCH, s.f.). Por su parte, la economía privada parte de la economía que busca el lucro en su actividad y que no está controlada por el Estado. Estas economías llevan a un equilibro económico a nivel nacional; promueven nuevas oportunidades de ingreso a los sectores al desarrollo de nuevos productos e innovación de modo tal que exista un equilibrio entre oferta y demanda para así evitar inflación. 
Durante el "Gran Foro Económico: ¿cómo afrontar los desafíos del 2019?" realizado en Bogotá por Foros Dinero y el Banco BBVA, mencionaron que "el país necesita enfrentar no solo las reformas coyunturales en materia fiscal sino un ajuste estructural en su economía (mejoramientos en sus planes de inversión y productividad) para direccionarlo a un crecimiento del 3.5\%" (Foros Semana, 2019). Para mejorar las condiciones de los sectores públicos, y privados, sigue discutiendo el Foro: "se necesitaría que para el 2019 el PIB creciera más allá del 5\% de lo que hasta el momento ha crecido lo que se podrían generar una mejor redistribución del ingreso y mayores oportunidades para todos los sectores" (Foros Semana, 2019).

Ahora bien, de acuerdo con datos estadísticos del Departamento Administrativo Nacional de Estadística (DANE) y a la Gran Encuesta Integrada de Hogares (GEIH):

Para el mes de enero de 2019 la tasa de desempleo fue 12,8\%, lo que representó un aumento de 1,0 \% respecto al mismo mes de 2018 (11,8\%). La tasa global de participación se ubicó en 63,5\% y la tasa de ocupación fue $55,3 \%$. En el mismo mes del año anterior estas tasas fueron $63,2 \%$ y $55,8 \%$, respectivamente (DANE, 2019, p. 3).

Los datos recogidos por el DANE muestran que el déficit del PIB para el año 2018 fue del 3.7\%; representa un mayor gasto de lo que ingresa al país. El gobierno propone que para el 2024 se baje ese porcentaje y llegue al 1\%, aceptable para la economía colombiana.

De acuerdo con La República: "se prevé que la inflación aumentará hasta 3,5\% para el 2019" (Monterrosa, 2018). Además, apunta que "los datos macroeconómicos muestran que en inflación el Índice de Precios al Consumidor (IPC) está ubicado en 3,27\% a noviembre, cifra que está dentro del rango meta del Banco de la República, que va de 2\% a 4\%". Si algo es seguro para este año 2019, tal como lo manifiesta El País:

Colombia espera muchos contrastes en la economía debido a la fuerte influencia del entorno mundial, los apremios fiscales del Gobierno y la incertidumbre que rodeará a variables como el dólar, el petróleo y las tasas de interés del sistema financiero. Otra variable que genera incógnitas es la inflación, ya que por cuenta del fenómeno de "el niño", podrían aumentar de manera significativa los precios de algunos alimentos, al reducirse la oferta en los mercados (Redacción El País, 2019).

Si se compara los dos entes que manejan y sostienen la economía en un país, como es el caso de Colombia, se puede evidenciar que a nivel 
macroeconómico, la calidad para los diversos procesos en productividad se puede llevar a niveles elevados, de acuerdo al resultado de PIB del año 2018. Lo anterior, parafraseando a Rodríguez Medina, Balestrini Solangel, Balestrini Sara, Meleán y Rodríguez Castro (2002) (En adelante Rodríguez Medina et al., 2002), arrastra a una tecnología más sistematizada en los procesos productivos; causa competencia en el mercado; a mayor calidad mayor costo de adquisición. Por ende, lleva a la industria a ofertar mayor producto sobrepasando la demanda, a tal punto de causar un desequilibrio entre oferta y demanda.

Microeconómicamente, el comportamiento de los agentes consumidores y productores pueden llegar a presentar en el año 2019 poca utilidad y beneficios bajos, de acuerdo con el año anterior. Se realiza esta aseveración debido a los indicadores de desempleo en aumento y al direccionamiento de los procesos de las grandes industrias hacia la tecnología y maquinaria para ser más eficientes frente a la competencia. Así, obligan a las empresas del sector microeconómico a impulsar sus procesos para estar acorde a lo exigido en dicha oferta y demanda

De la mano con lo anterior, la microeconomía no está en la capacidad de proveer la suficiente oferta, o grandes desarrollos; el nivel de acometividad y productividad no abarca el 100\% de lo exigido. Esto permite afirmar que en una economía donde hay desigualdad de oferta y demanda la tasa de desocupación aumenta cada vez, así que la falta de ingresos no permite un buen bienestar ni calidad de vida. En contraste con las grandes superficies, pertenecientes al sector privado, y parte de ligado macroeconómico, las cuales maximizan su beneficio, toda vez que al trabajar hacia el lucro ven como, no solo sus ganancias aumentan, sino que su economía es fluyente.

El economista Andrés Abadía, de la firma Phanteon Macroeconomics, en Londres, afirma que "el consumo privado probablemente será el principal impulsor del ciclo económico en Colombia una vez más, en consonancia con los mejores fundamentos internos, incluida la baja inflación, las bajas tasas de interés y un mercado crediticio resistente" (Reuters, 2019).

Con respecto a la afirmación del señor Abadía, el sector privado posiblemente podría llegar a impulsar la economía. Sin embargo, las nuevas reformas no se tienen en consentimiento, así como tampoco el impacto que pueden traer a los sectores. Desde un punto de vista consumidor, necesariamente los dos sectores son útiles para el movimiento de la economía y crecimiento del país; uno le da estabilidad y el otro impulso hacia los nuevos mercados. 


\section{Afectación de las nuevas TIC en el sistema económico colombiano}

Las nuevas TIC en el sistema económico colombiano tienen una capacidad de penetración en la sociedad de tal dimensión que genera todo tipo de impactos en la misma, así como repercusiones tanto económicas como sociales. En un país como Colombia, los impulsos que genera la innovación tienen efectos en el ámbito del conocimiento, así como en el desarrollo de nuevas tecnologías. Respecto al desarrollo de nuevas tecnologías, la misma es la causante, en gran medida, de la competitividad de los mercados.

Pero ¿qué sucede con la parte del "conocimiento" ?, lastimosamente la inversión en investigación y desarrollo que realiza el país es muy baja. Todavía no se ha caído en cuenta que:

En el mundo global, la mayoría de países considera fundamental la inversión en ciencia y tecnología por ser un factor clave para el crecimiento y desarrollo económico y constituirse como uno de los principales promotores de la productividad y competitividad, teniendo en cuenta que el conocimiento científico, ha liderado diferentes innovaciones en beneficio para la sociedad (Portafolio, 2017).

De acuerdo con el Observatorio Colombiano de Ciencia y Tecnología (OCyT), en el año 2016 la inversión en investigación, innovación y desarrollo estuvo alrededor del $0.27 \%$ del PIB. Esto coloca de manifiesto el rezago del país en este tipo de inversión; fundamental para la generación de nuevo conocimiento. No se ha podido entender que a través de la generación de conocimiento, se agrega valor a los recursos del país. Esto redundaría en mayores y mejores exportaciones, así como contar con una tecnología propia, acorde con los requerimientos del país.

Es importante resaltar que:

Aunque se ha destinado el $32 \%$ de los recursos de regalías para promover la investigación y desarrollo, fundamentales para resolver las problemáticas de la sociedad desde la investigación, se requiere de un fortalecimiento de las relaciones entre ciencia y sector productivo que garantice una transferencia efectiva de conocimiento para lograr un sector productivo más innovador, competitivo y productivo en el país (Portafolio, 2017). 
Aunado a lo anterior, y de acuerdo con Portafolio:

Colombia apenas invierte una décima parte del promedio de los países de la OCDE; menos de una octava parte de lo que invierte China, y una séptima parte de lo que invierten en promedio países de renta media alta a los cuales pertenecemos. Y nos encontramos no solo por debajo de Brasil, el líder latinoamericano en este campo, sino también de otros de la región (Argentina, Costa Rica, Cuba, Chile, Ecuador, México y Uruguay) (Otros Columnistas, 2018).

Coincide, entonces, con las duras observaciones realizadas por académicos y científicos en el denominado "Manifiesto de la Ciencia". El manifiesto es producto del trabajo llevado a cabo en Paipa, Boyacá, a comienzos del año 2018. Allí expresan: Carecemos de un verdadero sistema y de una política nacional de ciencia, tecnología e innovación (CTI) que incluya a todos los actores y establezca mecanismos de coherencia entre la política educativa, la industrial, y la de ciencia y tecnología. Esta política debería ir acompañada de recursos para un razonable desarrollo de la CTI (Redacción Vivir, 2018).

También mencionan que:

La falta de liderazgo de Colciencias, la poca inversión en ciencia y tecnología (que es poco más del 0.2\% del PIB nacional), y la manera deficiente en que consideran que funciona el Sistema General de Regalías en materia de Ciencia y Tecnología, "que en más de una ocasión, ha desalentado a la comunidad científica con proyectos que no tienen relación ni pertinencia alguna para el Sistema Nacional de Ciencia, Tecnología e Innovación (SNTI) (Redacción Vivir, 2018).

Lo anterior lleva a manifestar que el país requiere de una política en ciencia, tecnología e innovación, que no solo incluya a todos los sectores, sino que establezca mecanismos articuladores entre la política educativa, la industria, la ciencia, y la tecnología. Esto, en común acuerdo entre los actores involucrados, con el fin de no sufrir afectaciones en los desarrollos que traen, las nuevas industrias sistematizadas y los nuevos mercados donde todo funciona digitalmente.

En acuerdo a lo expuesto por José Ocampo Gaviria (2017), Codirector del Banco de la República: Colombia es uno de los países que en las nuevas tecnologías de la comunicación se ve afectado por su escasa participación tecnológica; el recurso de inversión en esta área realmente es nula, llevando al país a un ahogo constante, al no tener los recursos de innovación tecnológica, el sistema económico se ve en declive pues no tiene como sostener esta demanda y los mercados por ende se ven afectados desde varios aspectos, tales como: 
- Mano de obra poco calificada.

- Sistemas Productivos convencionales.

- Desarrollo económico dividido.

Lo anterior coloca sobre el tapete la necesidad asidua de que el tema de ciencia y tecnología ocupe un lugar preponderante en las agendas políticas del país, si lo que se quiere es llevar al país por un verdadero camino de crecimiento económico y social. El no hacerlo, como ha ocurrido hasta el momento, afecta, de manera negativa, a los sistemas productivos convencionales en Colombia, provocando una menor producción, lo que afecta de manera directa a la productividad.

Y si de productividad se habla, es importante anotar que muchas empresas colombianas le han apuntado a las nuevas tecnologías de la información y de la comunicación, para aumentar la misma. No es raro ver que las empresas colombianas han direccionado su enfoque empresarial al proceso de informatización, lo que ha cambiado el paradigma tecno productivo y de masificación de la producción.

Tanto para los países desarrollados como para los países en vías de desarrollo, se ha evidenciado que las TIC son la ficha fundamental para alcanzar los propósitos de los sectores económicos (globalización, mercados sistematizados, nuevas formas de producción), lo que llevará al crecimiento económico y desarrollo social-cultural.

Una de las soluciones que el gobierno podría implementar, es el brindar apoyo a cada sector económico, donde el recurso presupuestal este dirigido a: primero el cambio tecnológico; segundo, la implementación de nuevas formas de producción; tercero, altos estándares de calidad, y cuarto la combinación de la mano de obra con la nueva tecnología desde un punto estratégico para no afectar la economía de los trabajadores. Esto se logra a través de:

- Combinación máquina-hombre

- Adaptación de lo tradicional con la innovación técnica

- Sistematización de tiempo y recurso

- Capacitación del recurso humano

Desde un ámbito cultural este enfoque ayudaría a la revolución del país, al crecimiento y desarrollo, donde se puede brindar una comunicación técnica dentro de los nuevos sistemas tecnológicos favoreciendo la economía del país a nivel interno y externo. 


\section{Los nuevos requerimientos y retos del mercado laboral ante el uso de las tecnologías y las TIC}

Si bien es cierto, las TIC han traído beneficios tales como el desarrollo, el mejoramiento en la economía, el aumento en la calidad educativa, la inclusión, y el rompimiento de fronteras. También han originado que las organizaciones evolucionaran ante los nuevos requerimientos del entorno y han obligado a que las mismas generen mecanismos de adaptación y de respuesta.

Entre los nuevos requerimientos, a los que las organizaciones han tenido que hacer frente, se tiene la necesidad de contar con personal cualificado, a la vanguardia de la tecnología, con una visión prospectiva. Este ha sido uno de los retos más grandes, no solo a nivel organizacional, también a nivel académico; a las instituciones educativas les ha caído la mayor responsabilidad de formar profesionales competentes, que den respuesta a las necesidades del entorno.

No es de olvidar que en la actual época todo se vuelve asequible. Las TIC han generado un gran avance en todos los campos de la educación, la ciencia y la economía, independiente de sus problemáticas. Este nuevo acceso a la información brinda a las personas la posibilidad de conocer y aprender sobre lo que sea, en el momento que deseen. Además, cada día se debe preparar académicamente para los nuevos retos que traen los cambios tecnológicos. Así, es evidente el hecho del posicionamiento de la tecnología, llegando inclusive a poblaciones aisladas y vulnerables, desde ese punto de partida habla de inclusión.

En la actualidad, y gracias a la globalización, el mercado laboral exige a los profesionales una serie de competencias y habilidades orientadas hacia la comprensión y manejo de las TIC. Estas permiten a las empresas aumentar sus niveles de producción, mejorar la calidad del trabajo, y optimizar tiempos de respuesta, lo que repercute en una mayor eficiencia en el proceso. Para los empleados, esto se traduce en un óptimo desempeño de sus funciones; para los clientes, en una mejor relación con la empresa. Es evidente, las ventajas del uso de las TIC en una empresa, sin embargo, hay que tener en cuenta los desafíos para los empleados y empleadores; el principal de ellos: la continua capacitación de los profesionales, en especial de las áreas administrativas. No obstante, y en especial para los recién egresados, las TIC se han convertido en una buena forma de entrar al mundo laboral.

Ahora bien, se debe tener a consideración que las TIC no solo han transformado al mundo empresarial; su influencia tentacular ha llegado a los más recónditos lugares del mundo laboral; origina nuevas oportunidades de empleo, mayor innovación y mejora en la inclusión, y mayor competitividad, en los mercados laborales. 
Las TIC se han convertido en una forma de combatir el desempleo existente al interior de los países. Teletrabajo, aplicaciones en teléfonos móviles, internet, son apenas algunas de las estrategias laborales utilizadas por millones de personas alrededor del mundo. Como manifiesta el Banco Mundial (BM) (2013):

Las TIC conectan a las personas con puestos de trabajo. Los mercados laborales en Internet ayudan a unos 12 millones de personas de todo el mundo a encontrar trabajo vinculándolos con empleadores a nivel mundial. Babajob en India, Duma y M-Kazi en Kenya y Souktel en Oriente Medio y Norte de África son casos de servicios de búsqueda de empleo que usan herramientas móviles y basadas en la web. Estos logran que los mercados de trabajo sean más inclusivos. Por ejemplo, Souktel apunta a comunidades de bajos ingresos.

Además, sigue diciendo la entidad multilateral; "a hoy, y gracias a las TIC'S, se han generado nuevas formas de empleos, tales como: la contratación en línea y las plataformas de microtrabajos" (Banco Mundial, 2013). Aunado a lo anterior, se tiene que hoy en día, para la contratación de un empleado, debe tener como mínimo algunos de los siguientes requisitos:

- Conocer el manejo de un equipo o maquina.

- Tener conocimientos de programas básicos para el desarrollo laboral.

- Estar certificado en algún curso realizado acorde la necesidad de la labor.

- Haber trabajo en el campo al que se está aspirando a ser contratado.

Las tendencias del mundo laboral están en continuo cambio. Así como hay profesiones que han permanecido incólumes a través del tiempo (como la medicina) hay otras que atraen la atención de una gran cantidad de jóvenes, como aquellas relacionadas con la era digital, pese a ser nuevas. Es la tendencia del momento; brinda virtudes y cualidades para la competitividad laboral. De una cosa se debe estar seguro: no es posible, tan siquiera imaginarnos, a qué punto de evolución llegará el mundo laboral. Al respecto, y tal como indicó Julio César Saldarriaga, coordinador de Virtualidad de la Universidad de Antioquia, "...la demanda de profesionales relacionados con nuevas tecnologías este en crecimiento por la ventajas que esta trae (mejor perfil, educación actualizada, mayores facilidades de adquirir un trabajo, facilidad de adaptación en el mundo Digital)".

El avance de las TIC en un país como Colombia implica que la competencia entre mercados será mayor y los clientes serán más exigentes en cuanto a calidad y tiempo de respuesta por parte de las compañías proveedoras. 
Sin embargo, a medida que las expectativas de los clientes crecen, el presupuesto para TIC tiende a disminuir en la búsqueda de eficiencias productivas (sistemas versus recurso humano). Así, estas se enfrentan al reto de entregar más servicios a un menor costo y ahí es donde comienza a evidenciarse el desequilibrio entre los profesionales más experimentados con conocimientos frescos.

Las TIC han promovido no solo el cambio de vida tradicional de la sociedad; han revolucionado el estilo de vida ambiguo por uno vanguardista. Esto se hizo evidenciable en el momento en que todo empezó a realizarse en tiempo real, con información real, veraz, con facilidad de desarrollo e innovación. Esta es la razón por la que los profesionales deben estar actualizados en cuanto a los gustos de las compañías, así como a sus necesidades, y a lo que esperan de su equipo de trabajo. Pero también a lo nuevo que se viene en el mercado.

Los cambios en el mercado laboral son un desafío y una oportunidad, dependiendo de las personas que están expuestas a ellos. Es apreciable la creciente demanda por las profesiones de las ciencias administrativas, y por las profesiones de los sistemas de información. No es de extrañarse que en la actualidad se encuentren más especialistas y menos profesionales generalistas. La fuerza laboral actual combina diversas generaciones y no es raro encontrar trabajadores jóvenes, y mayores, en los mismos ramos, desempeñando cargos similares.

Por otra parte, hay que tener en cuenta que, según lo señala Luis Felipe Jaramillo, director del programa Negocios Internacionales de la Universidad de Medellín: "posiblemente algunas carreras desaparecerán y otras nuevas surgirán e incluso "las tradicionales apuntarán hacia otro lado" (Buitrago, 2018). Además, apunta:

La oferta educativa en las universidades tiene que ir de la mano con las necesidades del mercado laboral, por lo que las tendencias en las carreras que más se estudian cambian con el desarrollo de nuevas tecnologías y las modificaciones en las necesidades del mercado (Buitrago, 2018).

El experto, a su vez señala que:

Las carreras tradicionales como medicina, derecho y arquitectura siguen siendo muy estudiadas, pero eso no siempre se corresponde con el mundo laboral. Por ejemplo, "hay un número demasiado grande formado en ciencias jurídicas, más de lo que necesita el mercado, lo importante es la especialización, hay áreas que no son cubiertas, áreas no tradicionales como derecho económico, tributario o de telecomunicaciones (Buitrago, 2018). 
La anterior cita deja entrever que siempre habrá profesiones que aunque el entorno cambie, quedan incólumes (como es el caso de la medicina y sus ramas). Sin embargo, en el otro extremo están aquellas carreras que deben irse adaptando, perfeccionando, amoldando y evolucionando de manera obligada, conforme a los cambios acaecidos en el entorno. Las administración de empresas es una de ellas.

En estasus profesionales, si es que deseanser competitivos laboralmente, deben estar en continua actualización por el simple hecho de que la variación del mercado nunca será igual a la acaecida el día anterior; porque la moneda siempre tendrá afectaciones cambiantes, que, sin lugar a dudas, arremeterá al interior de las organizaciones; porque cada decisión que tome el gobierno en materia de política económica y social causará un remolino de circunstancias que afectará a nivel empresarial y productivo.

Esta realidad hace que las Instituciones de Educación Superior (IES) tengan la responsabilidad de actualizar sus planes de estudios; ajustarlos a la realidad y buscar que los estudiantes pertenecientes a las áreas administrativas estén a la altura de los requerimientos del mercado laboral. El objetivo: formar profesionales competitivos, capaces de enfrentarse sin miedos a un exigente mercado laboral y que entiendan la importancia de incorporar las TIC a su vida profesional y laboral.

Es de entender que las TIC Ilegaron para quedarse. Tanto así que el quehacer organizacional se ha amoldado entorno a las mismas. Además, se ha generado una dependencia de las TIC no solo a nivel de los procesos organizacionales, también de la sociedad entera. No por nada cuando se habla de TIC, no se está hablando de tecnología, en el sentido escueto de la palabra. En cambio, se habla de comunicación, información, educación y competitividad; engloba recurso humano, producción, oferta y demanda, es decir, se llega a hablar de desarrollo social, desarrollo cultural y desarrollo económico.

\section{Las nuevas TIC a nivel de los sistemas de producción flexible}

A partir de la Segunda Guerra Mundial, el mundo ve con escepticismo la emergencia de un nuevo orden económico, de matices liberales; lleva a que las principales economías globales transaccionaran de una economía industrial a una posindustrial. Esto trae como consecuencia una globalización de las estructuras de producción. El resultado: la entrada de la competitividad, y el surgimiento de China como superpotencia industrial. 
Las grandes empresas, caracterizadas por poseer un sólido músculo financiero, empiezan a dominar y a colocar las reglas de juego en el denominado mercado-producto. Esto origina la necesidad de encaminarse a nuevas formas de procesos de producción que cumplieran con dos (2) objetivos. Por un lado, conseguir una industria en gran escala de productos relativamente homogéneos, con facilidad de ganancia (fabricación en masa). Por otro lado, disminuir de manera ostentosa los gastos de producción.

Aunque al principio garantiza un consumo duradero y una oferta continua, con el pasar del tiempo el modelo Fordiano (producción en serie) comienza a deteriorarse por su inflexibilidad. Esto, gracias a que los consumidores empezaron a cambiar sus preferencias, sobresaliendo su pluralidad. Pluralidad que, de acuerdo con Jaime González Martínez (s.f.)

Se deriva de la saturación y la fragmentación de los mercados para los bienes estandarizados y producidos masivamente; por otro lado, la erosión de los ritmos crecientes de productividad, y en consecuencia, el estrangulamiento de las ganancias derivado del crecimiento del costo relativo del trabajo.

Así, se comienza a abrir el camino hacia la producción flexible. De manera progresiva inicia a generar innovaciones en los procesos de producción, llevando no solo al:

Redimensionamiento del tamaño de las plantas y la redistribución y reorganización de los equipos; la modificación en las formas de organización y división de los procesos de trabajo orientados a intensificar el consumo productivo de la fuerza de trabajo y a la eliminación de inventarios, productos en proceso y desechos, el cambio de normas en las relaciones con los proveedores y consumidores son algunos elementos que dieron un sentido distinto al modelo de producción antiguo (González, s.f.).

Los grandes emporios productivos empezaron a hacer uso de las tecnologías; se fueron incorporando en el proceso de producción, desde el input hasta el output. El resultado: una automatización productiva que trajo consigo mayores índices de producción. Esto, a su vez, aumentó la exigencia, para el mercado laboral, en cuanto al manejo, uso y adecuación de las TIC. Entonces, se buscaba construir cadenas de suministro, completamente integradas, adaptables dinámicamente a las necesidades del consumidor en tiempo real y a la demanda de los proveedores y los consumidores en sentido creciente; todo a un bajo costo para incrementar la economía corporativa de la empresa. 
Esto incluyó la capacidad de anticiparse y tomar medidas correctivas en producción en tiempo real (procesos, tiempos, insumos calidad). Lo anterior generó una transformación revolucionaria, en términos de crecimiento, así como una automatización del proceso de producción, lo que aminoró la fuerza de trabajo. De aquí que las TIC se han vuelto parte fundamental de las esferas socioeconómicas del país.

Al minarse la fuerza de trabajo, y al adecuar el proceso productivo a las condiciones cambiantes del entorno económico, se abrió las puertas al crecimiento corporativo. Esto, toda vez que la automatización provocara la generación de mayores ingresos en las organizaciones, a un bajo costo, manteniendo la calidad exigida por el consumidor final. Esto hizo que se entrara en el terreno de la competitividad, terreno en el cual juegan las empresas que actúan conforme a las exigencias del entorno, pero que a su vez tienen en cuenta las preferencias del cliente. Así, a través de la diversificación, se posicionan en nichos de mercados y generan reglas de juego desde las fuerzas del mercado Porterianas.

\section{Conclusiones}

Colombia representa un punto estratégico para algunos países inversionistas; toda vez que es un país con grandes recursos sin explotar, y en proceso de desarrollo entre las masas. Es importante tener en cuenta que el gobierno tiene la capacidad de apoyar al sector público; si bien no se encamina hacia el lucro, tiene importantes connotaciones en el bienestar social. No se ha logrado entender que el crecimiento económico viene de la mano del desarrollo social.

Por otro lado, la complejidad del desafío en aceptación de la nueva tecnología frente a la cuarta Revolución Industrial lleva a que las compañías sean cada día más exigentes en cuanto a la mano de obra a contratar. Hay que tenerse en cuenta que cada pequeño detalle cuenta para que la economía sea efectiva.

Finalmente, la capacidad de las TIC en la producción actual no solo ha modificado sus parámetros internos y externos, también ha ofrecido capacidad de que sus sistemas computarizados se actualicen y se reformen. Además, provocan que las empresas, además de tener mejores oportunidades en el mundo productivo, puedan ser competitivas, tenga un buen flujo de dinero, y estén a la vanguardia de los cambios económicos y competitivos que traen los nuevos mercados. 


\section{Lista de referencias}

Banco Mundial. (2013). Conectarse para trabajar: Cómo las TIC amplían las oportunidades de empleo en todo el mundo. Banco Mundial. Recuperado de: https://www.bancomundial.org/es/news/feature/2013/09/10/howicts-are-expanding-job-opportunities

Buitrago, A. (16 de septiembre de 2018). El mercado laboral cambia con las TIC. ElMundo.com. Recuperado de: https://www.elmundo.com/ noticia/El-mercado-laboral-cambia-con-las-TIC/374641.

\section{Constitución Política de Colombia [Const.] (1991)}

Escobar, M. (20 de mayo de 2019). El sector privado colombiano: motor del desarrollo. La República. Recuperado https://www.larepublica.co/ especiales/las-1000-empresas-mas-grandes-de-2018/el-sector-privadocolombiano-motor-del-desarrollo-2863898, Revisado el 15/06/2019

Famuch. (s.f.). Economía Social. Famuch. Recuperado de: http:// famuch.org/economia-social/

Foros Semana. (2019). Gran Foro Económico ¿Cómo afrontar los desafios del 2019? Semana. Recuperado de: https://www.forossemana. com/evento/id/34731/gran_foro_economico_como_afrontar_los_desafios_ del_2019

González, J. (s.f.). De producción en serie a producción flexible. Comunicación Paradigmática Wiki. Recuperado de: https://comunicacinparadigmtica.fandom.com/wiki/De_producci\%C3\%B3n_en_serie_a_ producci\%C3\%B3n_flexible

Mojica Montoya, F. (2018). Los retos del talento humano en el conflicto. Bogotá: Editorial Fundación Universitaria San Mateo. Recuperado de https://palma.sanmateo.edu.co/

Monterrosa, H. (30 de diciembre de 2018). Conozca las cifras económicas con las que arranca el próximo año. La República. Recuperado de: https://www.larepublica.co/economia/conozca-las-cifras-economicascon-las-que-arrancara-el-proximo-ano-2810494

Otros Columnistas. (12 de agosto de 2018) El atraso colombiano en ciencia y tecnología. Portafolio. Recuperado de: https://www.portafolio. co/opinion/otros-columnistas-1/el-atraso-colombiano-en-ciencia-ytecnologia-519977 
Portafolio. (18 de junio de 2017). Las regalías y la inversión en ciencia y tecnología en Colombia. Portafolio. Recuperado de: https://www.portafolio. co/economia/las-regalias-y-la-inversion-en-ciencia-y-tecnologia-encolombia-506940

Redacción El País. (06 de enero de 2019). Así se moverán los motores de la economía colombiana en el 2019. El País. Recuperado de: https://www. elpais.com.co/economia/asi-se-moveran-los-motores-de-la-economiacolombiana-en-el-2019.html

Redacción Vivir. (06 de junio de 2018). Cámara de Representantes aprueba proyecto para crear Ministerio de Ciencia, Tecnologías e Innovación. El Espectador. Recuperado de: https://www.elespectador. com/noticias/ciencia/camara-de-representantes-aprueba-proyecto-paracrear-ministerio-de-ciencia-tecnologia-e-innovacion-articulo-792803

Reuters. (24 de febrero de 2019). Expertos esperan un crecimiento de 2.67\% para el 2018. Portafolio. Recuperado de https://www. portafolio.co/economia/expertos-esperan-un-crecimiento-de-2-67-parael-2018-526744

Rodríguez M. Et al. (2002). Análisis estratégico del proceso productivo en el sector industrial. Revista de Ciencias Sociales (Ve), VIII(1), pp. 135-156. Recuperado de: https://www.redalyc.org/articulo.oa?id=280/28080109 